\title{
On the Effect of Heterogeneous Traffic Sources on the Network Availability for Wireless Sensor Grids
}

\author{
Ali Hammad Akbar ${ }^{1}$, Ki-Hyung Kim ${ }^{1, *}$, Shaokai $\mathrm{Yu}^{2}$, \\ and Won-Sik Yoon ${ }^{3}$ \\ ${ }^{1}$ Graduate School of Information and Communication \\ ${ }^{2}$ Ajou University (Invited Faculty Programme) \\ ${ }^{3}$ School of Electrical Engineering \\ Ajou University, Suwon, Korea, 443-749 \\ \{hammad, kkim86, wsyoon\}@ajou.ac.kr, \\ yshaokai@yahoo.com
}

\begin{abstract}
Wireless sensor grids are poised for applications where predeployment information is highly available to optimize communication and computation costs. Using two widely known grid traversal schemes, we critique the effect of multiple services being rendered by a sensor network onto its availability. We derive an interesting result using Markovian model to appreciate the effect of load heterogeneity onto node and network lifetimes. Our mathematical model also analyzes synchronous sleep for its effect on energy conservation. We also propose a epicenter regulated, asynchronous sleep scheme which allows nodes to conserve energy yet relay data towards the sink effectively. The performance results show that the proposed scheme prolongs network availability in sensor grids.
\end{abstract}

\section{Introduction}

Wireless sensor networks are unique in the sense that they are supposed to work unattended. For their longevity, network designers propose schemes that reduce the computation and communication model. The fact that sensor networks have a growing application in telematics, home control, and ubiquitous services is a relief to sensor network community, wherein protocol designs are simple yet efficient.

In wireless sensor grids, pre-deployment information is much useful to the designers. They can trim heavy and cumbersome protocols of general purpose sensor networks to have simpler equivalents by exploiting location awareness.

One such kind of information is the type of traffic that flows over the sensor grid [1]. For non-real time services, traffic flow is highly adjustable. Vice versa, real time services' traffic flows have high variance due to greedy approach and resulting contention. Network availability as identified in [2] provides researchers with a baseline to diagnose and regulate node and network parameters for enhancing operational lifetime. In [3], the authors suggest a scheme for optimal transmission range distribution to balance out power consumption across sensor nodes as a linear programming

\footnotetext{
* Corresponding author.
} 
problem. They propose a numerical model that gives upper bounds on energy inefficiencies due to energy balancing strategies. Likewise, in [4], Yang et al. give a dichotomy power control mechanism for prolonging network availability. They split the sensor network region of interest (ROI) into concentric rings. An algorithm then provides rules for adjusting transmission power such that all nodes dissipate energy evenly.

In this paper, we analyse sensor grids [5] for gauging the effect of traffic heterogeneity onto their longevity. In [6], we have already derived network availability expression of sensor grids for two widely known lattice path traversal that handle just a singular traffic type.

The contribution of this paper is to visualize network longevity from the application-centric viewpoint. We appreciate the innate nature of today's heterogeneous services i.e., real and non real time services. We also critique the notion that traffic generated sporadically versus a heavy network load has a multi-dimensional relationship with the longevity of a network. Furthermore, we propose a sleep schedule for sensor grids that is cognizant of the kind of traffic sensor network supports. Our algorithm allows real-time applications to make the choice of source-dependent sleep schedules or as spelled out by the sink. Non real-time applications conveniently allow the sink to announce sleep schedules. The performance results validate the strength of our proposal when combined with carrier sensing on the link layer. Our design considerations throughout the paper befit well with the state-of-the-art in silicon technology, such as sleep modes of sensor nodes, and in software e.g., TinyOS®.

\section{Communication Model and Fundamental Assumptions}

Fig. 1 illustrates a sensor grid that supports heterogeneous services viz real and nonreal time services. For the sake of simplicity, the services are classified as both real time and non real time services. Following assumptions are made to formulate the model;

- Some sensor nodes generate real time data such as tracking information for military applications. It is typical example of a push model. Here traffic shaping is impossible.

- Other sensor nodes generate non real time data. A buffer, again shown in Fig. 1, gives an allowance to the network to shape the traffic in order to avoid collisions. First-in-first-out and drop tail are the queuing and scheduling mechanism enforced.

- Carrier sensing such as MACA is supported.

- Unicast communication is adopted. Link layer information such as remaining battery of one hop neighbours can determine the right of way for the next node to transmit, amongst the downstream neighbours.

- $h_{i j}$ is the initial energy of the sensor node $(i, j)$ at the reference time $t_{0}$, distributed across the network as $\eta_{i j} e^{-\eta_{i j} t}$ with mean $1 / \eta_{i j}$.

- Sensing processes, both for real and non real-time applications are temporally independent We assume Poisson distribution with mean $\lambda_{i j}$ [7]. We further denote this variable with subscripts $r$ (real time) and $n r$ (non real-time) to distinguish between the two traffic types. 
- Energy consumptions due to these activities are exponentially distributed with mean $1 / \beta_{i j}$ and $1 / \gamma_{i j}$ respectively.

- Sensor node $(i, j)$ consumes energy at a rate of $a_{i j}$ per second to remain awake.

- Sensor node $(i, j)$ consumes energy at a rate of $a_{i j}^{\prime}$ per second during the time they sleep. While a node is sleeping, it does not perform relaying for other nodes' data. For the sake of simplicity in analytical modeling, we consider a sleeping node to behave the same as a dead node-especially in the context of routing. The dead node creates holes that result into network partitions.

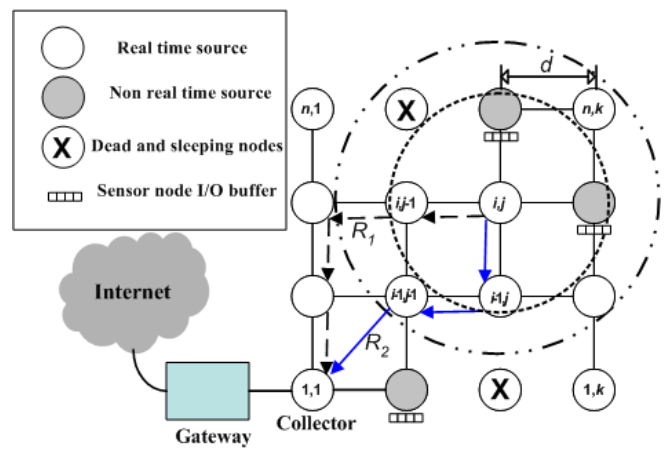

Fig. 1. A sensor grid with heterogeneous traffic sources

\section{Numerical Analysis of Network Availability}

We denote $y_{i j}$ as the total energy consumed by sensor node $(i, j)$ in sensing, relaying, during sleep, and in idle mode. Furthermore, $x_{i j}$ denotes energy consumption just being sensing and relaying data only. $F_{t}\left(x_{i j}\right), \forall i j=\{11, \ldots, 1 k, 21, \ldots, 2 k, \ldots n 1, \ldots, n k\}$ is the joint probability density function (pdf) of all nodes at time $t$. Since Poisson processes are pure birth processes, the joint pdf of all the sensor nodes can be given by the differential-difference equation as

$$
\begin{aligned}
& \frac{d F_{t}}{d t}\left(x_{i j}\right)=-\sum_{i=1}^{n} \sum_{j=1}^{k}\left(\lambda_{i j_{r}}+\lambda_{i j_{n r}}\right) F_{t}\left(x_{i j}\right)+\sum_{i=1}^{n} \sum_{j=1}^{k}\left(\lambda_{i j_{r}}+\lambda_{i j_{n r}}\right)\left(\beta_{i j_{r}}+\beta_{i j_{n r}}\right) \\
& \times \prod_{l=1}^{i-1} \prod_{m=1}^{j-1}\left(\gamma_{l m_{r}}+\gamma_{l m_{n r}}\right) \int_{y_{11}=0}^{x_{11}} \ldots \int_{y_{i j}=0}^{x_{i j}} F_{t}\left(y_{11}, \ldots, y_{i j}, \ldots, x_{n k}\right) \times \\
& \exp \left[-\left(\beta_{i j_{r}}+\beta_{i j_{n r}}\right)\left(x_{i j}-y_{i j}\right)-\sum_{l=1}^{i-1} \sum_{m=1}^{j-1}\left(\gamma_{l m_{r}}+\gamma_{l m_{n r}}\right)\left(x_{l m}-y_{l m}\right)\right] d y_{11} \ldots d y_{i j} .
\end{aligned}
$$

Equation (1) reflects overall energy consumption for two kinds of traffic; real time and non real time. Considering (1) to be an initial value problem, we obtain $R_{t}\left(s_{i j}\right)$ as Laplace transform of $F_{t}\left(x_{i j}\right)$ : 


$$
R_{t}\left(s_{i j}\right)=\exp \left[-\left(\sum_{i=1}^{n} \sum_{j=1}^{k}\left(\lambda_{i j_{r}}+\lambda_{i j_{m}}\right)-\sum_{i=1}^{n} \sum_{j=1}^{k}\left(\lambda_{i j_{r}}+\lambda_{i j_{m r}}\right) \frac{\left(\beta_{i j_{r}}+\beta_{i j_{u r}}\right)}{s_{i j}+\left(\beta_{i j_{r}}+\beta_{i j_{v r}}\right)} \prod_{l=1}^{(i-1)} \prod_{m=1}^{(j-1)} \frac{\left(\gamma_{l m_{r}}+\gamma_{l m_{u r}}\right)}{s_{l m}+\left(\gamma_{l m_{r}}+\gamma_{l m_{u r}}\right)} \times t\right] .\right.
$$

In this paper, we analyze a synchronous sleep mode as identified in [8] for sensor nodes on detecting no activity. The total energy consumption of entire sensor network is now characterized by a single node's energy consumption, i.e., $y_{i j}=x_{i j}+a_{i j} t_{1}+a^{\prime}{ }_{i j} t_{2} ; t_{1}$ and $t_{2}$ being the awake and sleep times respectively and that $t=t_{1}+t_{2}$. Let $Z_{t}\left(s_{i j}\right)$ be the Laplace transform of $F_{t}\left(\mathrm{y}_{i j}\right)$.

$$
Z_{t}\left(s_{i j}\right)=R_{t}\left(s_{i j}\right) \times \exp \left[\sum_{i=1}^{n} \sum_{j=1}^{k} a_{i j} s_{i j} t_{1}+\sum_{i=1}^{n} \sum_{j=1}^{k} a_{i j}^{\prime} s_{i j} t_{2}\right] .
$$

We define henceforth, $A_{t}$ as the network availability-the probability that all the nodes along all the paths are alive. Inserting (2) into (3) and manipulating the variables, the network availability is given by

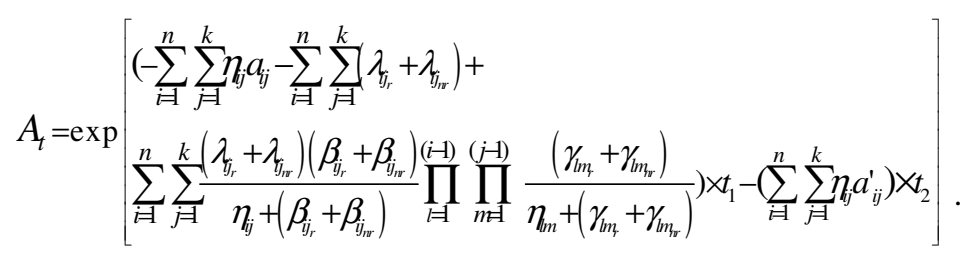

Considering two unicast data relaying models, i.e., Delannoy number-based and staircase lattice path traversals, we analyze the effect of traffic load and traffic heterogeneity on the network availability. If the transmission range is adjusted to $R_{1}$ as shown in Fig. 1, staircase lattice links $(\leftarrow \boldsymbol{\Downarrow})$ are used en-route to relay data from the sensing node to the gateway, and assuming a square topology, (4) can be transformed as

$$
A_{t_{S}}=\exp \left\{-\left[n^{2} \eta a+n^{2}\left(\lambda_{r}+\lambda_{n r}\right)-\frac{\left(\lambda_{r}+\lambda_{n r}\right)\left(\beta_{r}+\beta_{n r}\right)}{\left(\eta+\overline{\beta_{r}+\beta_{n r}}\right)} \times D\right] \times t\right\}
$$

where

$$
\left.D=\sum_{i=0}^{n-1}(1+i)\left(\frac{\overline{\gamma_{r}+\gamma_{n r}}}{\gamma_{r}+\gamma_{n r}}+\eta\right)\right)^{i}+\sum_{j=1}^{n-1}(n-j)\left(\frac{\overline{\gamma_{r}+\gamma_{n r}}}{\gamma_{r}+\gamma_{n r}+\eta}\right)^{(n-1)+j}
$$

Similarly, adjusting the power levels to transmission range of $R_{2}$, the data relaying activity turns out to be a different lattice path traversal $(\leftarrow \boldsymbol{\Downarrow})$, as given by Delannoy numbers [6]. The network availability of (4) is now given as

$$
A_{t_{D}}=\exp \left\{-\left[n^{2} \eta a+n^{2}\left(\lambda_{r}+\lambda_{n r}\right)-\frac{\left(\lambda_{r}+\lambda_{n r}\right)\left(\beta_{r}+\beta_{n r}\right)}{\left(\eta+\overline{\beta_{r}+\beta_{n r}}\right)}\left(\sum_{i=0}^{n-1}(2 i+1)\left(\frac{\overline{\gamma_{r}+\gamma_{n r}}}{\overline{\gamma_{r}+\gamma_{n r}}+\eta}\right)^{i}\right)\right] \times t\right\}
$$

The parameters in (5) and (6) are all assumed to be independent of $i, j, l, m$, and the traffic type, i.e., $a_{i j}=a, a_{i j}^{\prime}=a^{\prime}, \lambda_{i j}=\lambda, \beta_{i j}=\beta, \eta_{i j}=\eta, \eta_{l m}=\eta$, and $\gamma_{l m}=\gamma$. Numeric values are 
adopted from [9]: $a=15 \mu \mathrm{J} / \mathrm{s}, a^{\prime}=1.5 \mu \mathrm{J} / \mathrm{s}, \lambda=0.083$ packets per second, $1 / \eta=$ $12960 \mathrm{~J}, 1 / \beta=42.61 \mathrm{~J}, 1 / \gamma_{S}=140.87 \mathrm{~J}$ for staircase and $1 / \gamma_{D}=280 \mathrm{~J}$ for Delannoy. The number of nodes varies from 4 (or $n=2$ ) to 1600 (or $n=40$ ).

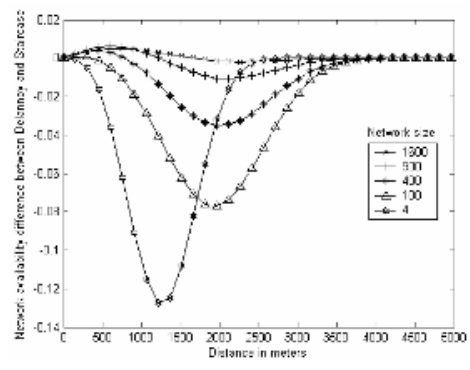

(a) Varying inter-node distance

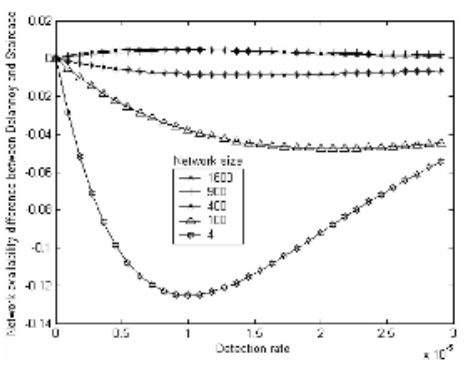

(b) Varying traffic of all sensor nodes

Fig. 2. Difference between network availabilities under topology size and traffic load variation

Fig. 2 (a) is the difference between (6) and (5) to show the effect of inter-node distance variation onto network availability. It was observed at $t=1000000$ seconds (11.57 days). The grid size was varied with a step size of $150 \mathrm{~m}$. When the inter-node distance is increased for a fixed number of sensor nodes, staircase is a better choice for relaying until the network size becomes exceedingly large, for which Delannoy performs better.

Fig. 2 (b) is the difference between (6) and (5) to show the effect of traffic load and heterogeneity onto network availability. For medium to very large networks, e.g., for networks with nodes exceeding 1600, Delannoy offers better performance for increasing traffic load. For heterogeneous sources, Delannoy is poised to given a performance gain as for real-time traffic, diagonal paths are available for immediate data delivery. For delay-flexible traffic staircase equalises in performance.

\section{Traffic Type-Cum-Load Aware Sleep Schedules for Sensor Grids}

For the sake of simplicity in numerical modelling, we adopted synchronous sleep mode for the sensor grids. To capture the behaviour of sensor grid under asynchronous sleep schedule (sensing fidelity is relatively higher here) is NP-hard problem. Since such sleeping nodes do not participate in relaying activity [8], more sleeping nodes contribute to frequent path failures. Energy conservation through sleep mode is compromised by packet loss and retransmissions. In order to assign sleep schedules to sensor nodes, three considerations must be taken into account

I) Total number of paths available for a node to relay data. It can be computed by

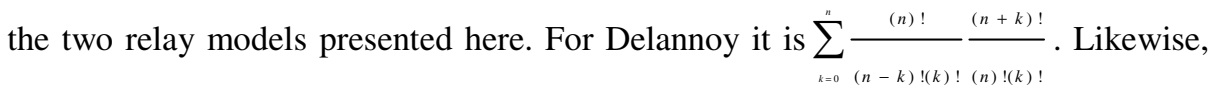
for staircase it is $\frac{(2 n-2) !}{(n-1) !(n-1) !}$. 
II) The total relay load a node carries. For a node $i j$, upto $(n-i+1)(n-j+1)-1$ nodes relay data through it. If the nodes adopt asynchronous sleep mode, and sleeping probability of each node is $P_{s}$, the minimum probability that data from node $(i, j)$ will be delivered successfully is given by $P_{D}=\left(1-P_{s}\right)^{[i+j-2]}\left(P_{s}\right)^{[i \times j-(i+j)-2]}$.

III) Type of traffic and data. For two kinds of traffic and their impact onto sleep schedules, it is important to consider their effects first in isolation and then in association to other factors such as upstream nodes and relay load. E.g., for real time applications, data loss is compensated through retransmissions, while for non real time traffic, buffering can be exploited too.

Non real time applications however fit well with sleep schedules spelled out by the sink such that every node is mindful of the sleep schedule of its downstream neighbours. Real time applications may also be given a leeway to make local decisions.

\subsection{Deterministic Sleep Mode Adoption}

When a node selects a downstream relay agent, it registers with that agent through a registration request. This control packet is a notification of letting the downstream neighbour know of a prospective sender. Additional information such as start time, whether real time or non real time traffic, and expected duration may also be specified here. Registration process continues till the epicenter. The epicenter compiles and propagates a final record of all the applications for which it will relay the data. This compilation is subsequently used by the node to adjust its sleeping schedule as described through Fig 3.

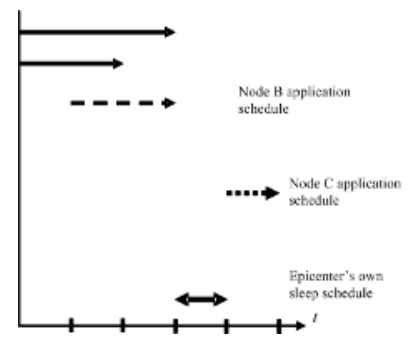

Fig. 3. Epicenter computes sleep schedule and shares with one hop neighbours

\section{Performance Analysis}

A simulator based on the system in section IV was developed in $\mathrm{C}++$ to evaluate our scheme.

Fig. 4 (a) illustrates simulation results obtained for staircase lattice path traversal under the three scenarios as shown in the legend. The advantage using our scheme is clear. Nodes closer to the gateway either do not sleep or sleep less. Thus they do not loose any routing data, resulting into network-wide energy saving when compared with uniform sleeping scheme and no sleep at all. 


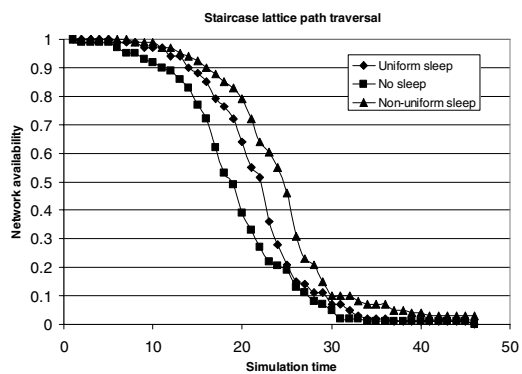

(a) Staircase lattice path traversal

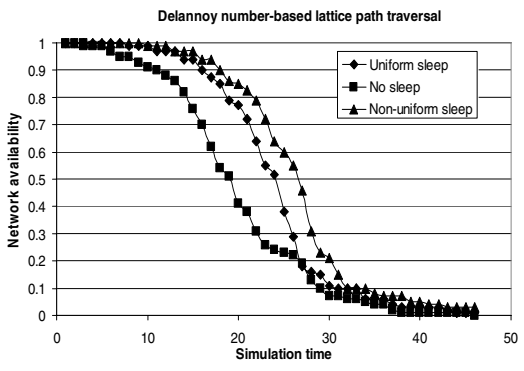

(b) Delannoy number-based path traversal

Fig. 4. Network availability comparison for sleep schemes

Fig. 4 (b) shows the comparison and simulation results asserting the usability of nonuniform sleep scheme in Delannoy number-based traversal. A fractional gain in network availability is observed compared to staircase traversal for all the three scenarios.

\section{Conclusion}

In this paper, we study wide range of parameters for wireless sensor grids. In particular, we focus on the relaying activity and traffic type for network longevity. We conclude that Delannoy performs better for real time applications by providing an additional path per hop. It also provides a gain in network availability due to reduction in hops for very large networks.

We propose a non-uniform, sleep scheme that is aware of the traffic load type of upstream neighbours. The paradox that nodes closer to the gateway sleep less yet enhance overall network connectivity and longevity is an important finding. The performance results show that the proposed scheme prolongs network availability effectively in sensor grids.

\section{Acknowledgement}

This research is supported by the ubiquitous Autonomic Computing and Network Project, the Ministry of Information and Communication (MIC) 21st Century Frontier R\&D Program in Korea.

\section{References}

1. Akyildiz, I.F., Weilian S., Sankarasubramaniam, Y. Cayirci, E.: A survey on sensor networks. IEEE Communications Magazine, IEEE, vol. 40, (2002), 102 - 114

2. Hiroshi, S., Hiroya, M.: Performance Issues and Network Design for Sensor Networks. IEICE Transactions on Communications, vol. E87-B (2001), 294-301 
3. Perillo, M., Zhao C., Heinzelman, W.: On the problem of unbalanced load distribution in wireless sensor networks. IEEE GLOBECOM, (2004), 74-79

4. Yang Z., Zhao D., Cheng W., He J.: Prolong the lifetime of wireless sensor network with dichotomy power control.: IEEE TENCON, (2004), 231-234

5. Shakkottai, S., Srikant, R., Shroff, N.: Unreliable sensor grids: coverage, connectivity and diameter. IEEE INFOCOM, vol. 51, no.12, (2002), 1448-53

6. Akbar, A. H., Yoon, W. S., and Kim, J. H.: Effect of Transmission Power Adjustments on Network Availability. Information Technology Journal, 4(3), 2005 271-273

7. Kleinrock, L.: Queuing Systems Volume I: Theory. Cambridge University Press (1997)

8. Tao Z., Radhakrishnan, S., Sarangan, V.: PMAC: An Adaptive Energy-Efficient MAC Protocol for Wireless Sensor Networks. IEEE Parallel and Distributed Processing Symposium, (2005), 65-72

9. Bhardwaj, M., Garnett, T., and Chandrakasan, A. P.: Upper Bounds on the Lifetime of Sensor Networks. in Proceedings of ICC, (2001), 785-790 